\title{
Disentangling orbital and spin textures of surface-derived states in non-symmorphic semimetal HfSiS
}

\author{
Xiaoxiao Wang (®), ${ }^{1}$ Jiahua Chen, ${ }^{1}$ Mingtian Zheng, ${ }^{1}$ Tatiana V. Menshchikova, ${ }^{2,3}$ Igor P. Rusinov, ${ }^{2,3,4}$ Eike F. Schwier, ${ }^{5}$ \\ Filip Orbanić, ${ }^{6}$ Shilong Wu, ${ }^{1}$ Kazuki Sumida, ${ }^{1}$ Tomoki Yoshikawa, ${ }^{1}$ Koji Miyamoto, ${ }^{5}$ Munisa Nurmamat, ${ }^{1}$ Taichi Okuda, ${ }^{5}$ \\ Kenya Shimada, ${ }^{5}$ Mario Novak, ${ }^{6}$ Evgueni V. Chulkov, ${ }^{3,4,7,8}$ and Akio Kimura ${ }^{1, *}$ \\ ${ }^{1}$ Graduate School of Science, Hiroshima University, 1-3-1 Kagamiyama, Higashi-Hiroshima 739-8526, Japan \\ ${ }^{2}$ Tomsk State University, 634050 Tomsk, Russia \\ ${ }^{3}$ Donostia International Physics Center (DIPC), 20018 San Sebastián/Donostia, Basque Country, Spain \\ ${ }^{4}$ Saint Petersburg State University, 198504 Saint Petersburg, Russia \\ ${ }^{5}$ Hiroshima Synchrotron Radiation Center, Hiroshima University, 2-313 Kagamiyama, Higashi-Hiroshima 739-0046, Japan \\ ${ }^{6}$ Department of Physics, Faculty of Science, University of Zagreb, Bijenička 32, 10000 Zagreb, Croatia \\ ${ }^{7}$ Departamento de Física de Materiales UPV/EHU, 20080 Donostia-San Sebastián, Basque Country, Spain \\ ${ }^{8}$ Institute of Strength Physics and Materials Science, Russian Academy of Sciences, 634021 Tomsk, Russia
}

(Received 3 April 2019; revised manuscript received 15 August 2019; published 27 November 2019)

\begin{abstract}
We have investigated the spin polarization and orbital configuration of the spin-split surface states in a nonsymmorphic semimetal HfSiS by spin- and angle-resolved photoelectron spectroscopy using polarized tunable photons combined with detailed theoretical calculations. We have found that the surface states surrounding the $\bar{X}$ point with $C_{2 v}$ symmetry exhibit an exotic unidirectional spin texture coupled with $d_{y z}$ orbital. The observed unidirectional spin texture is well reproduced by the theoretical calculations. Our work clarifies the significance of orbital symmetry in the manipulation of spin orientations, providing an approach to realize the persistent spin texture for the prolonged spin-coherence time in spintronic applications.
\end{abstract}

DOI: $10.1103 /$ PhysRevB.100.205140

\section{INTRODUCTION}

Recently extensive studies have been done on materials with strong spin-orbit coupling (SOC) because they may exhibit quantum phenomena originating from nontrivial topological properties of the electronic structures [1,2]. Furthermore, via strong SOC, the electron spin can be manipulated by the electric field, attracting many interests from the viewpoint of potential applications to spintronics as well as information technology [3-6]. One of the challenges for the spintronics application is to prolong the spin-coherence time. This can be achieved by creating a persistent spin texture (PST), where the spin orientation is independent of the momentum and maintains a quasi-one-dimensional spin configuration [7-15]. PST has been found in semiconductor heterostructures equalizing the Rashba and Dresselhaus spin-orbit contributions by external electric field $[10,11]$. However, the PST realized by a fine tuning of the external electric field has rather limited practical applications. Alternative ideas to realize PST without applying an external electric field have been proposed for (1010) surfaces of the wurtzite group of crystals with an in-plane electric polarization, and in the strained spin-split $\mathrm{LaAlO}_{3} / \mathrm{SrTiO}_{3}$ interfaces [16-18]. Very recently, the possibility of PST was also predicted in noncentrosymmetric bulk material $\mathrm{BiInO}_{3}$ and $\mathrm{SnTe}$ (001) oriented film with in-plane ferroelectricity $[19,20]$.

Recently the non-symmorphic Dirac nodal-line semimetals $W H M(W=\mathrm{Zr}$, Hf, La; $H=\mathrm{Si}, \mathrm{Ge}, \mathrm{Sn}$, and $\mathrm{Sb} ; M=\mathrm{S}$, Se,

*Corresponding author: akiok@hiroshima-u.ac.jp
Te, O) [21-29] have been theoretically and experimentally investigated. HfSiS is the most representative compound because of the emergence of spin-split surface states (SSs) due to strong SOC at the $\bar{X}$ point with $C_{2 v}$ symmetry near the Fermi level $[22,24]$. Thus, HfSiS could be a good candidate to realize exotic orbital and spin texture due to spin orbital fields on the surfaces. However, until now, there has been no systematic investigations on the orbital configuration and spin texture of these surface states.

In this study, we have directly investigated the orbital and spin configurations of the surface-derived states in HfSiS by means of spin- and angle-resolved photoelectron spectroscopy (SARPES) employing variable linearly polarized light as well as theoretical calculations within density functional theory. We demonstrate that the spin texture of the SSs is unidirectional, namely, the spin direction is almost parallel to the $\bar{\Gamma} \bar{X}$ line irrespective of the $\boldsymbol{k}$ point and that the spin texture is originated from the modulation of the orbital texture and can also be reproduced by considering equal contributions from the Rashba and Dresselhaus spin-orbit coupling terms. Because of the ease in fabrication, environmental friendliness, and easy accessibility to the PST configuration, $\mathrm{HfSiS}$ is a promising candidate for applications in spin transistors and spin-logic circuits with the potential to enhance substantially spin coherence time [30].

\section{EXPERIMENT AND METHODS}

High quality single crystals of $\mathrm{HfSiS}$ were grown by the chemical vapor transport method described elsewhere 
(a)
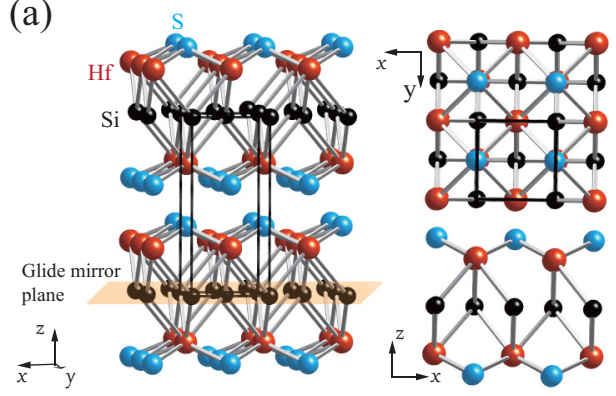

(b)

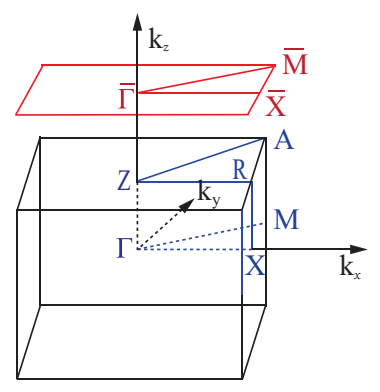

(c)

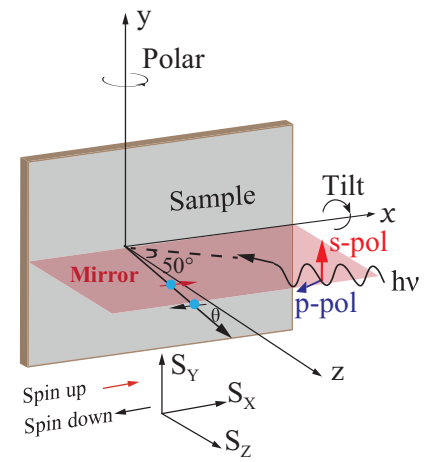

(d)
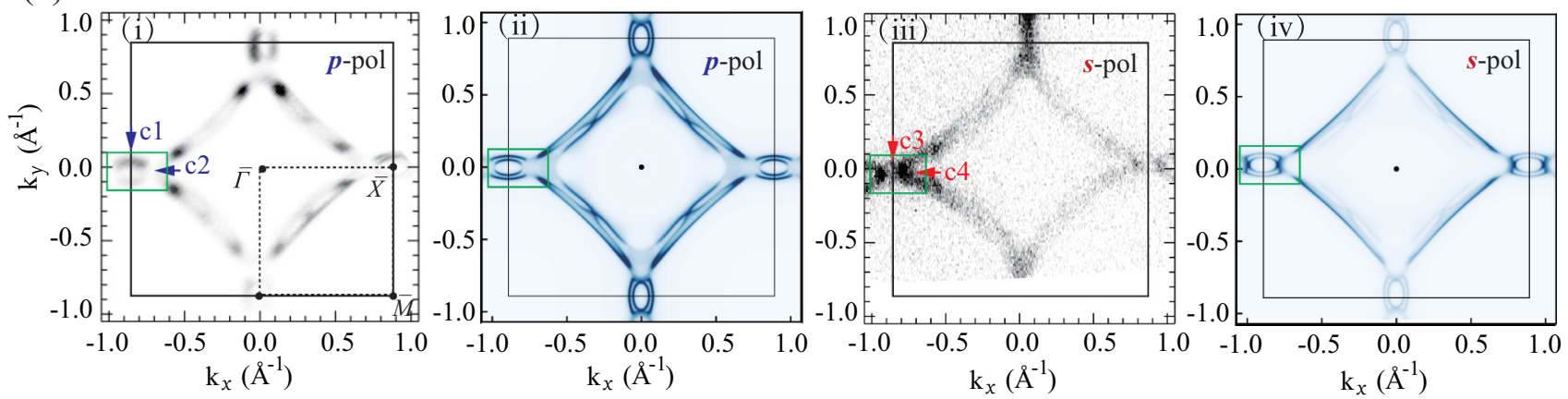

FIG. 1. (a) Crystal structure of HfSiS with $P 4 / n m m$ symmetry. The glide mirror plane is colored orange; top and side views of the lattice structure are given on the right. (b) Bulk Brillouin zone and projection onto the (001) surface. (c) Experimental configuration: the $x$ - $z$ plane is a mirror plane. $S_{X}-S_{Y}-S_{Z}$ are the spin coordinates. (d) Experimental Fermi surface measured at $h v=60 \mathrm{eV}$ in $p$-polarization geometry (i) and $s$-polarization geometry (iii). Blue and red arrows denote cuts (c1, c2, c3, c4) for the measured ARPES band dispersion in Figs. 2(a) and 2(b). The $\bar{\Gamma} \bar{X}\left(\boldsymbol{k}_{\boldsymbol{x}}\right)$ line is parallel to the laboratory $x$ axis [Fig. 1(c)]. (ii) and (iv) Corresponding calculated Fermi surfaces for the $p$ - and $s$-polarization geometries, respectively.

[31]. Angle-resolved photoelectron spectroscopy (ARPES) measurements with changeable linearly polarized light with a photon energy of $60 \mathrm{eV}$ were performed at BL-1 of the Hiroshima Synchrotron Radiation Center (HSRC) [32]. Based on the Fermi's golden rule, the photoelectron intensity is in proportion to the square of the matrix element $\left\langle\phi_{f}|\boldsymbol{A} \cdot \boldsymbol{P}| \phi_{i}\right\rangle$ for the dipole transition, where $\left|\phi_{i}\right\rangle$ and $\left|\phi_{f}\right\rangle$ are the initial and final states, respectively, and $\boldsymbol{A}$ and $\boldsymbol{P}$ are the incident photon vector potential and the electron momentum operator, respectively [32,33]. Note that $\boldsymbol{A}$ is parallel to the electric field vector $\boldsymbol{E}$ of incident photon. In the present experiment, we set the incidence plane of $100 \%$ linearly polarized undulator radiation to coincide with the detection plane of the electron energy analyzer. By rotating the electron analyzer around the incident light axis, we can switch the vector potential parallel to the incidence plane ( $p$-polarization geometry: $\boldsymbol{A} \cdot \boldsymbol{P}$ is even symmetric with respect to the detection plane) or perpendicular to the incidence plane ( $s$-polarization geometry: $\boldsymbol{A} \cdot \boldsymbol{P}$ is odd symmetric with respect to the detection plane). Here we assume that the final state $\left|\phi_{f}\right\rangle$ is free-electron-like being even symmetric with respect to the detection plane. By setting the mirror plane of the sample to the detection plane (= incidence plane), the matrix element is exactly zero for the initial state $\left|\phi_{i}\right\rangle$ being odd symmetric with respect to the mirror plane in the $p$-polarization geometry. On the other hand, the matrix element is exactly zero for $\left|\phi_{i}\right\rangle$ being even symmetric with respect to the mirror plane in the $s$-polarization geometry.
Therefore, one can selectively observe even or odd symmetric initial states by changing the polarization geometry.

SARPES measurements were performed at $h v=24$ and $26 \mathrm{eV}$ using the high-resolution SARPES apparatus (efficient spin-resolved spectroscopy observation machine: ESPRESSO [34]) installed at beamline BL-9B of the HSRC. The typical energy and momentum resolutions were $10 \mathrm{meV}$ and $0.1^{\circ}$, respectively. The high efficiency of the very low energy electron diffraction spin polarimeters enables us to perform the SARPES measurements with high energy and angular resolutions $\left(E \sim 20 \mathrm{meV}, \theta \sim \pm 0.35^{\circ}\right)$. The experimental data were collected under ultrahigh vacuum conditions at a pressure below $6 \times 10^{-11}$ Torr. The effective Sherman function of the detector was 0.22. Samples were cleaved in situ around $30 \mathrm{~K}$ under a vacuum condition better than $6 \times 10^{-11}$ Torr at both beamlines. The in-plane $\left(S_{x}, S_{y}\right)$ and out-of-plane $\left(S_{z}\right)$ spin components of the sample can be obtained by the spin-detector system. The difference between the original spin polarizations in the sample coordinate system and the projected spin component onto the spin-detector coordinate system are considered [35,36].

Electronic structure calculations were carried out using the projector augmented wave method [37,38] implemented in VASP [39]. The exchange-correlation energy was treated using the generalized gradient approximation with the PerdewBerke-Ernzerhof functional [40]. The Hamiltonian contained scalar relativistic corrections, and the SOC was taken into 

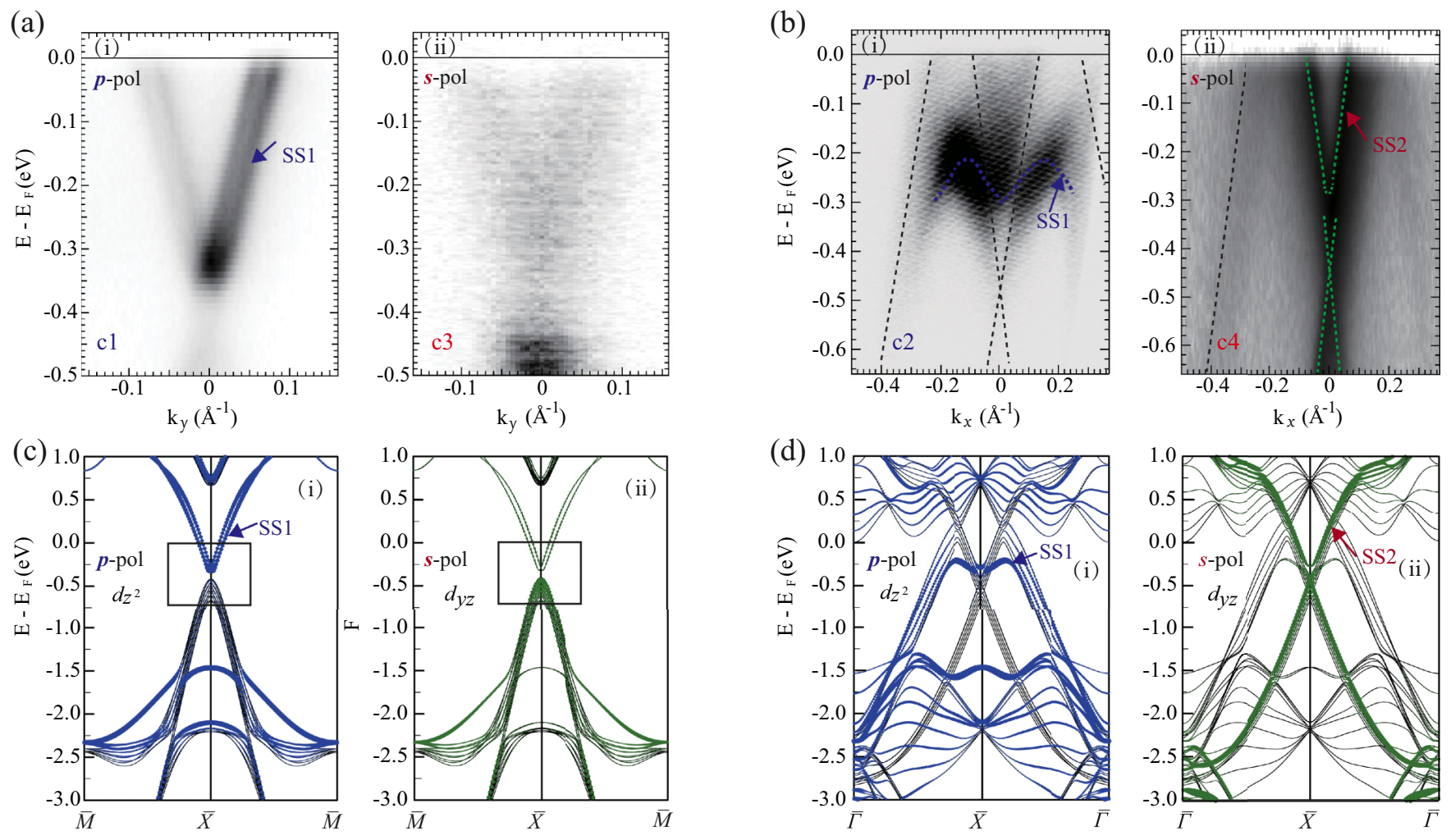

FIG. 2. (a) and (b) Measured energy-band dispersions along the $\bar{M} \bar{X}$ and $\bar{\Gamma} \bar{X}$ lines, respectively. The black and green dotted lines are visual guides for the bulk and surface bands. (c) and (d) Calculated orbital-resolved band structures corresponding to (a) and (b). The size of the colored circles indicates magnitudes of the $d_{z^{2}}$ or $d_{y z}$ orbital weight in the energy bands. The blue and red arrows point to the surface states SS1 and SS2, respectively.

account by the second variation method [41]. To calculate the (001) surface electronic structure and to prevent finite size effect we use symmetric 30-atomic layer slab with $12 \AA$ of separating vacuum. Constant energy contours were calculated within recursive green function technique $[42,43]$ on top of the linear combination of pseudo atomic orbitals approach as realized in the OPENMX code [44].

\section{RESULTS AND DISCUSSION}

Figures 1(a) and 1(b), respectively, show the crystal structure and Brillouin zone (BZ) of HfSiS. The band structure is characterized by space group $P 4 m m$ (or point group $C_{4 v}$ ) at the $\bar{\Gamma}$ point and $P m m 2$ (or point group $C_{2 v}$ ) at the $\bar{X}$ point. To observe electronic states in detail we study the Fermi surface map (FSM) of HfSiS [Fig. 1(d)] using the $p$ - and $s$-polarization geometries shown in Fig. 1(c). The prominent feature in the FSM is the olive-shaped electron pocket around the $\bar{X}$ point [marked by the green rectangle in Fig. 1(d) (i)]. Considering the experimental geometry and the matrix elements for the dipole transition (see Fig. 1(c) and Ref. [31]), one can observe initial-state orbitals having even symmetry with respect to the $k_{x}-k_{z}$ mirror plane $\left[p_{x}, p_{z}\right.$, $d_{x z}, d_{x^{2}-y^{2}}, d_{z^{2}}$, see Fig. 1(c)] in the $p$-polarization geometry, whereas only initial states having odd symmetry are excited $\left(p_{y}, d_{x y}, d_{y z}\right)$ in the $s$-polarization geometry. As seen from Fig. 1(d), the shape and distribution of the spectral weight in FSM around the $\bar{X}$ point are significantly different for the $p$ - and $s$-polarization geometries indicating clearly directional alternation of the orbital symmetry.

The calculated Fermi surface spectral function for the $p$ and $s$-polarization geometries [Fig. 1(d) (ii) and (iv)] matches very well the experimental results. Our analysis shows that the Hf-derived $d_{x^{2}-y^{2}}, d_{z^{2}}$, and $d_{x z}$ orbitals contribute to the spectral weight mainly along the $\bar{\Gamma} \bar{X}$-line when the $p$-polarized geometry is used (see Fig. 1(c) and Fig. S1 of Supplemental Material [45]). In contrast, the data acquired with the $s$-polarization geometry clearly highlights additional bands being part of the SSs with the odd $p_{y}, d_{y z}$, and $d_{x y}$ orbitals (Fig. 1(c) and Fig. S1 of the Supplemental Material [45]).

To understand further the electron orbital configuration, we focus on the high-symmetry lines in the BZ. We find that the Rashba-like spin split SSs along the $\bar{M} \bar{X}$-line (indicated as SS1) [Fig. 2(a) (i)] appear in the $p$-polarization geometry. While under the $s$-polarization geometry [Fig. 2(a) (ii)], the Rashba-like surface spectral weight is strongly suppressed, indicating the even orbital character. As can be seen in Fig. 2(c), the density functional theory calculations of the surface band structure result in a good agreement with the measured data. Additionally, analysis of the orbital composition shows that the main contribution to SS1 corresponds to the $d_{z^{2}}$ orbitals (see Fig. S2 in Supplemental Material for details [45]).

In Fig. 2(b) (i), the band dispersion along the $\bar{\Gamma} \bar{X}$ line shows a Dirac-electron-like band dispersion in the $p$-polarization geometry. The M-shaped dispersion around $-300 \mathrm{meV}$ is connected with previously observed SS1 along 
(a)

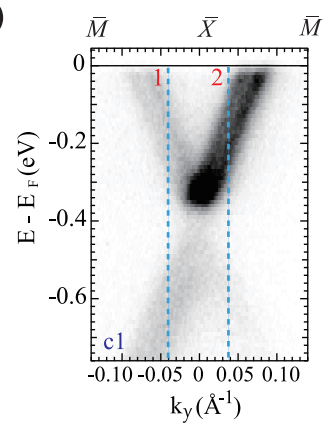

(c)

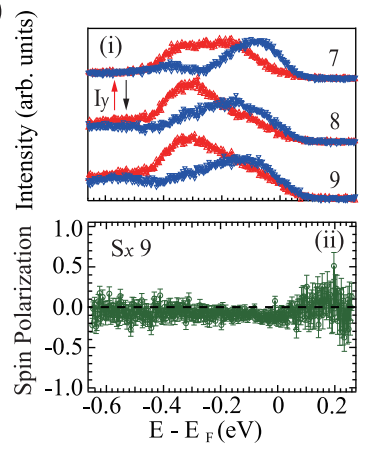

(b)
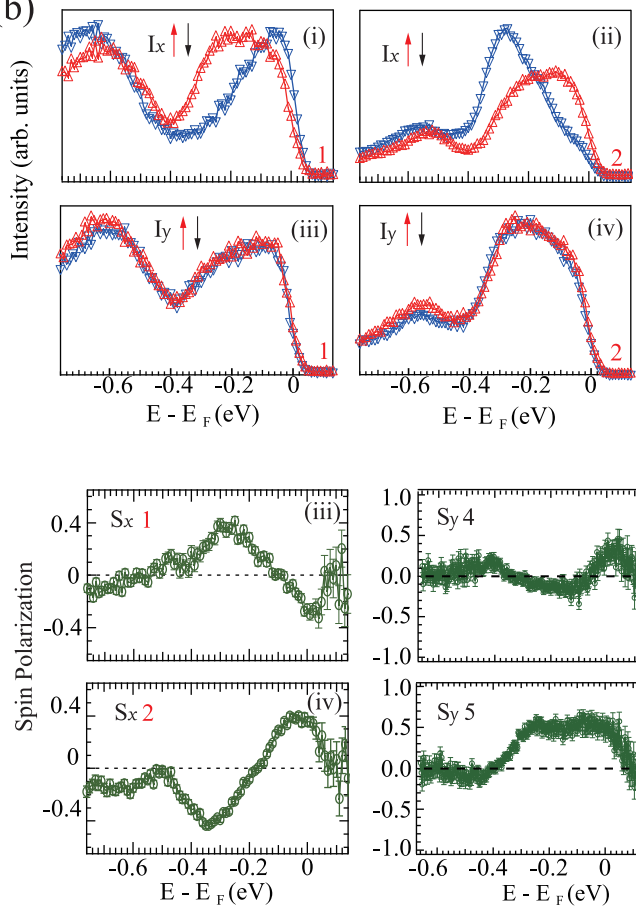
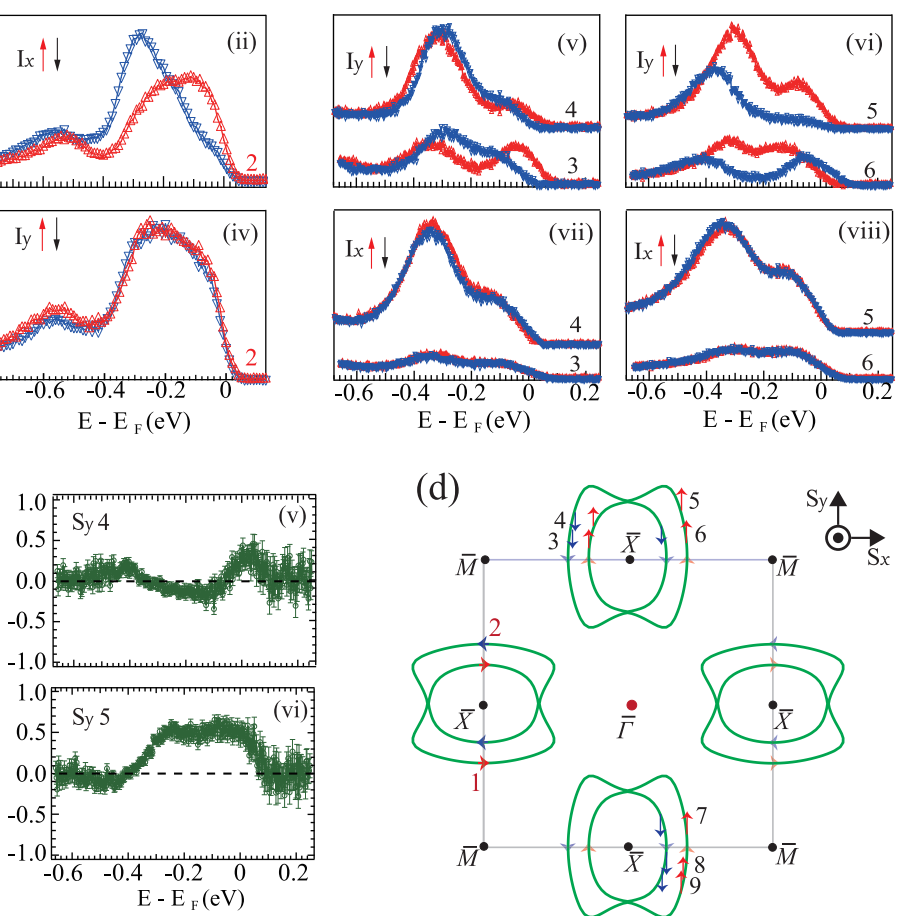

(d)

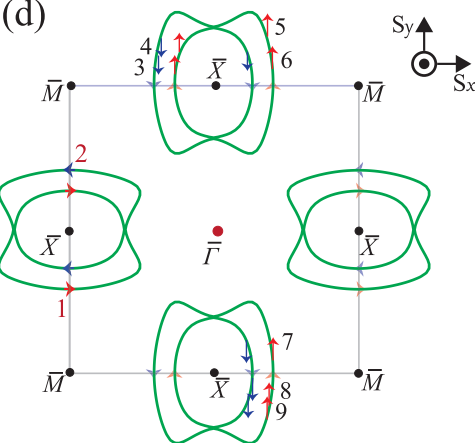

FIG. 3. (a) ARPES dispersion intensity maps of HfSiS with p-polarized light acquired with a photon energy (hv) of $24 \mathrm{eV}$ along the $\bar{M} \bar{X}$-line [c1 in Fig. 2(a)]. (b) SARPES energy distribution curves (EDCs) (red and blue lines show the spin-up and spin-down EDCs), and the wave vector positions of the spin-resolved EDCs [Figs. 3(a) and 3(d)]. (c) (i) Spin-resolved EDCs for the spin components along the $y$ direction measured at the positions marked 7-9 in Fig. 3(d) and below is the corresponding net $x$ component of spin polarization for cut 9. Right is the net $x$ component of spin polarization for cut1 (2) and $y$ component for cut 4 (5). (d) Schematic figure of the spin texture of the surface-derived electron pocket based on the experimental results.

the $\bar{M} \bar{X}$ direction and emerges from the bulk states at lower binding energies. In contrast, the ARPES result for the $s$ polarization geometry [(Fig. 2(b) (ii)] exhibits a surface state (SS2) which has a steep dispersion and forms the arc-like Fermi surface piece of the electron pocket around the $\bar{X}$ point [Fig. 1(d) (iii) and (iv)]. We conclude that SS2 is dominated by odd-symmetry orbitals, and according to the theoretical calculations such orbitals mainly correspond to the $d_{y z}$ type [Fig. 2(d)]. Consistent with its behavior along the $\bar{M} \bar{X}$ line, the spectral weight of the M-shaped SS1 almost vanishes along the $\bar{\Gamma} \bar{X}$ line in the $s$-polarization geometry. All these results clearly indicate that SS1 and SS2 are distinct states which are formed by orbitals with opposite symmetries (even or odd).

After identifying the orbital character of the surfacederived electron pocket, we also find that SS1 state has intriguing orbital resolved spin texture. To analyze the spin polarization of the SS1 by SARPES, we plotted the spin-up (I $\uparrow$ ) and spin-down (I $\downarrow$ ) energy-distribution curves (EDCs) and the related spin polarization measured at the different positions of the electron pockets around the $\bar{X}$ points in Figs. 3(b) and 3 (c). Here we examine spin-resolved EDCs along the $\bar{M} \bar{X}$ line (i.e., $k_{y}$ direction) with $k_{y}$ position $-0.04 \AA^{-1}$ (labeled as 1) and $+0.04 \AA^{-1}$ (labeled as 2) in Fig. 3(a). In Figs. 3(b) (i and ii), the EDCs with in-plane spin components $(x)$ exhibit reversed spin polarizations with the opposite $k_{y}$ momenta with respect to the $\bar{X}$ point, giving clear evidence of a Rashba-type contribution in the spin splitting of the SS1. As expected from symmetry-reflection arguments, in Figs. 3(b) (iii and iv), the spin polarization is negligible for the $y$ component for cuts 1 and 2 [Figs. 3(a) and 3(d)].

To scrutinize the spin character of this surface-derived electron pocket (SS1), we have also measured SARPES for the upper and lower electron pockets around the $\bar{X}$ point at the positions marked 3-9 in Fig. 3(d). The measured spin-resolved EDCs for the $y$ spin component for cut 3 (4) [Fig. 3(b) (v)] and cut 6 [Fig. 3(b) (vi)] on the inner and outer branch of the upper electron pocket show reversed spin direction. Interestingly, the $x$ spin component for cuts 3-6 were found to be negligible [Fig. 3(b) (vii and viii)], which supports a unidirectional spin texture with nontangential spin orientation being distinct from the conventional Rashba-type spin texture. Noticeably, label 5 on Fig. 3(d) is the $\boldsymbol{k}$ point on the outer branch close to the corner of the electron pocket, which shows the largest spin polarization for cuts labels 1-6 [see in Fig. 3(b) (v) and Fig. 3(c) (iii, vi)]. Furthermore, the spin-resolved EDCs for the $y$ spin component for the cuts labels 7-9 shows the same spin direction [Fig. 3(c) (i)] as in those of the cuts 5 and 6 , while negligible $x$ spin component was further confirmed as shown in Fig. 3(c) (ii). Thus, we verify an exotic unidirectional spin texture and clarify the whole spin texture of the four electron pockets as shown in Fig. 3(d), where the spin orientation rotates clockwise remaining independent of the momentum as one travels along 
(a)

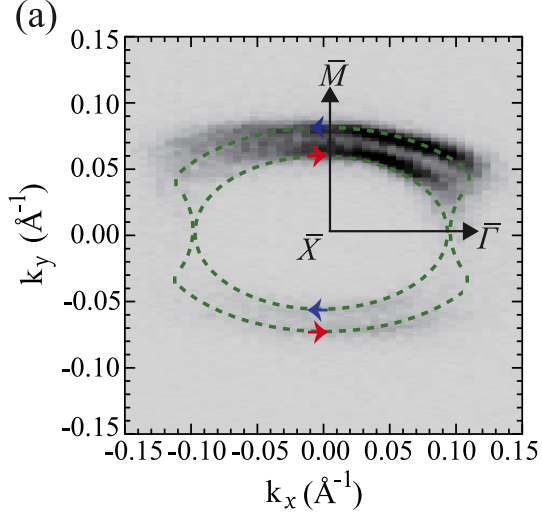

(d)

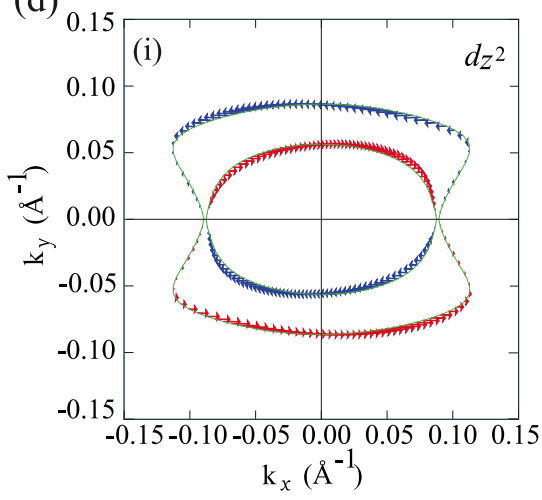

(b)
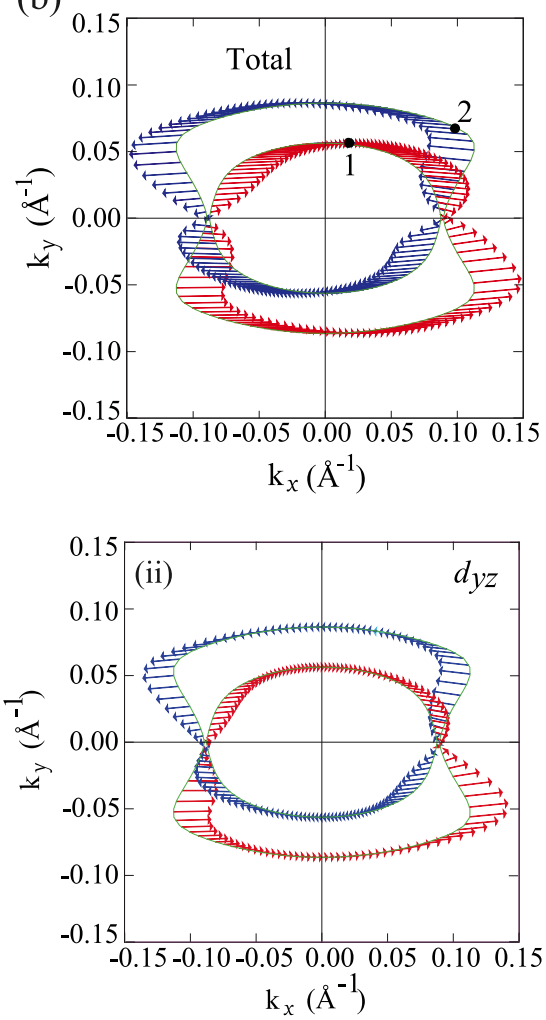

(c)
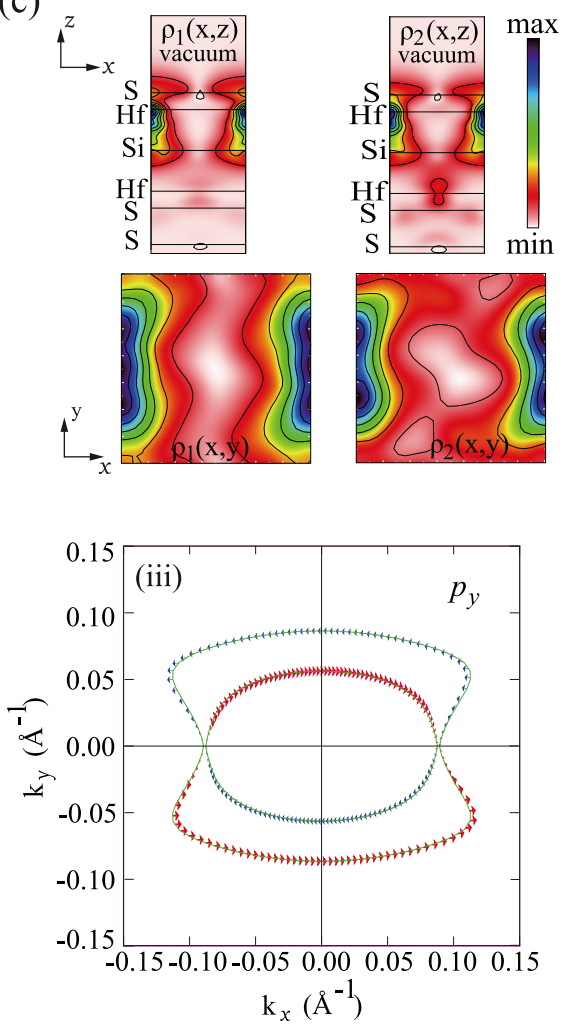

FIG. 4. (a) Sketch of the $\bar{X}$ pocket with spin directions near the $\bar{M} \bar{X}$-line deduced from SARPES data. (b) Corresponding theoretical total spin texture of the surface-derived electron pocket at the Fermi level; Arrows indicate both the total spin magnitude and direction, the blue and red coloring indicating different spin directions. (c) $\rho_{1}$ and $\rho_{2}$ projected charge distribution of the surface states onto the $x-z$ and $x-y$ plane at the $\boldsymbol{k}$ positions, respectively, labeled as 1 and 2 in panel (b). (d) Calculated orbital-resolved spin configuration.

the inner branch, but counterclockwise for the outer branch for all the electron pockets around the $\bar{X}$ point.

Figure 4(a) shows experimentally observed Fermi surfaces around the $\bar{X}$ point. The distinct Fermi surfaces where two contours have opposite spin direction and shifted with respect to each other in momentum space are well reproduced by our theoretical calculations [Fig. 4(b)]. Theoretical spin texture analysis of these states reveals dominant $S_{x}$ contribution with the absence of the $S_{y}$ spin components [Fig. 4(b)]. The spin texture distinctly shows almost no changes in the spin orientation, and the spin polarization reaches the largest magnitude around the corner of the electron pocket, which are in complete accord with our experimental results. Furthermore, we find that the calculated persistent spin texture is coupled with the orbital symmetry as shown explicitly for the tangential spin character with $d_{z^{2}}$ exactly along the $\bar{M} \bar{X}$ line, while maintaining a uniform spin orientation configuration imposed by the $d_{y z}$ orbital component [see Fig. 4(d) in detail]. Evidently, this orbital modulation in the spin texture is fully consistent with our linear-polarized light experimental results.

The resulting spin texture in HfSiS cannot be described within classical Rashba Hamiltonian model. To explain the observed features, we start with the charge density distribution of the SSs over the $x-z$ and $x-y$ planes at the $\boldsymbol{k}$ points marked as 1 and 2 in Fig. 4(b). The charge density distribution over the $x-z$ plane for both positions show strongly localized SSs within the outermost trilayer block with maximum on the Hf atom [Fig. 4(c) upper panel]. The charge density vanishes gradually in the bulk and is quite anisotropic along the $z$ direction introducing an out-of-plane local electric field. Such effective electrostatic potential gradient in the direction perpendicular to the surface of HfSiS is the origin of the "conventional" Rashba spin-orbit contribution. More importantly, charge density is also characterized by in-plane anisotropy [Fig. 4(c) lower panel].

Since the HfSiS surface possesses $C_{2 v}$ point group symmetry at the $\bar{X}$ point, we employ the $\boldsymbol{k} \cdot \boldsymbol{p}$ Hamiltonian to get further insight into the spin-split SSs at nonzero $\boldsymbol{k}$ (with respect to the $\bar{X}$ point). The SOC Hamiltonian possessing $C_{2 v}$ symmetry for SSs near the $\bar{X}$ point has two linear contributions:

$$
\begin{aligned}
\hat{H}(\boldsymbol{k}) & =\alpha_{1} \sigma_{x} k_{y}+\beta_{1} \sigma_{y} k_{x} \\
& =\alpha_{R}\left(\sigma_{x} k_{y}-\sigma_{y} k_{x}\right)+\alpha_{D}\left(\sigma_{x} k_{y}+\sigma_{y} k_{x}\right),
\end{aligned}
$$

where $\alpha_{R}=\frac{1}{2}\left(\alpha_{1}-\beta_{1}\right)$ and $\alpha_{D}=\frac{1}{2}\left(\alpha_{1}+\beta_{1}\right)$ describe the respective strengths of the Rashba contribution and of the $C_{2 v}$ warping (Dresselhaus) term because of the nonequivalence of the two in-plane directions. In the special case of $\alpha_{R}=\alpha_{D}$ (see Fig. 5), one obtains $\alpha_{1}=2 \alpha_{R}\left(=2 \alpha_{D}\right)$ and $\beta_{1}=0$, and the remaining term $\alpha_{1} \sigma_{x} k_{y}$ produces the unidirectional spin polarization [46]. Thus, the proposed spin-orbit Hamiltonian model with equally strong Rashba contribution and $C_{2 v}$ warping term allows us to reproduce the calculated and the observed unidirectional spin texture. 

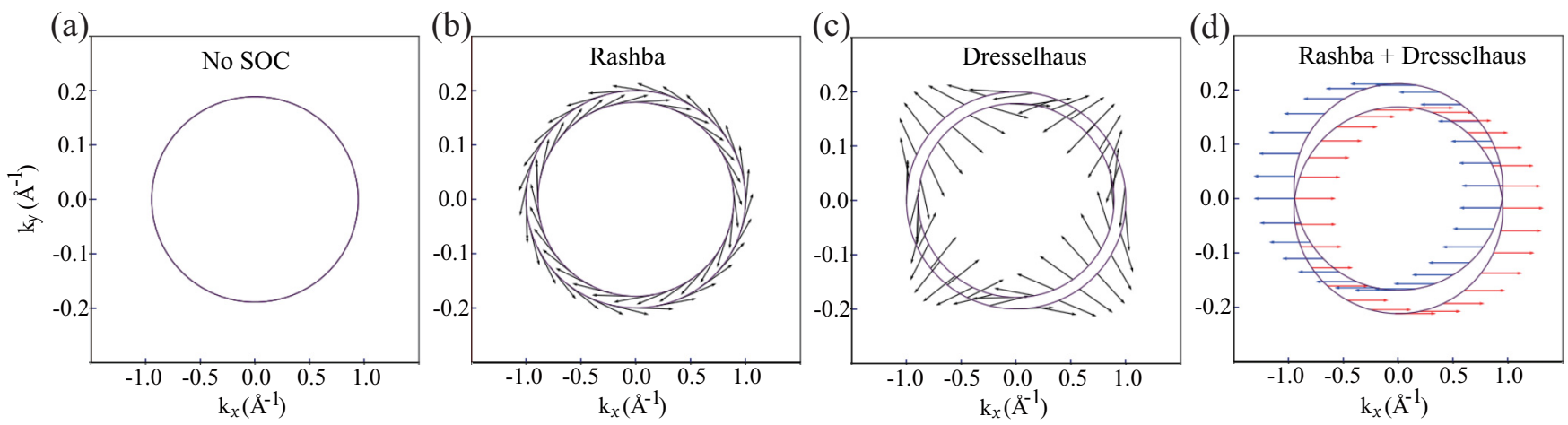

FIG. 5. Calculated spin textures of two-dimensional free electron system using $\boldsymbol{k} \cdot \boldsymbol{p}$ Hamiltonian with $C_{2 v}$ symmetry for (a) $\alpha_{R}=\alpha_{D}=0$, (b) $\alpha_{R} \neq 0, \alpha_{D}=0$, (c) $\alpha_{R}=0, \alpha_{D} \neq 0$, and (d) $\alpha_{R}=\alpha_{D} \neq 0$.

\section{CONCLUSIONS}

To summarize, spin and orbital characteristics of the spinsplit SSs in HfSiS have been confirmed by spin- and angleresolved photoelectron spectroscopy with variable light polarizations. First, we clarified that the SSs are mainly composed of $d_{z^{2}}$ along the $\bar{M} \bar{X}$ line that switches to $d_{y z}$ along the $\bar{\Gamma} \bar{X}$ line. More importantly, we confirmed a unique unidirectional spin texture mainly linked to the $d_{y z}$, which is also well reproduced by the $a b$ initio calculation. The combination of the Rashba and Dresselhaus spin-orbit interaction terms with equal contribution reasonably explains this unidirectional spin texture and can be applied for further theoretical investigation of materials with similar properties. Our result suggests that orbital symmetry linked to the spin orientation is crucially important for the formation mechanism of the persistent spin texture that provides a direction to prolong the spin coherence time through the manipulation of the orbital symmetry in the layered non-symmorphic materials.

\section{ACKNOWLEDGMENTS}

This work was financially supported by KAKENHI (Grants No. 17H06138, No. 17H06152, and No. 18H03683). The experiments were performed with the approval of the
Proposal Assessing Committee of HSRC (Proposals No. 18AG038, No. 17BG029, No. 17BG028). K. Sumida and T. Yoshikawa were financially supported by the Grant-in-Aid for JSPS Fellows No. 19J00858 and No. 18J22309, respectively. M. Novak and F. Orbanić acknowledge the support of the Croatian Science Foundation under the project (IP-2018-01-8912) and CeNIKS project cofinanced by the Croatian Government and the EU through the European Regional Development Fund - Competitiveness and Cohesion Operational Programme (Grant No. KK.01.1.1.02.0013). We acknowledge the support by the Basque Departamento de Educación, UPV/EHU (Grant No. IT-756-13), Spanish Ministerio de Economia y Competitividad (MINECO Grant No. FIS2016-75862-P), Fundamental Research Program of the State Academies of Sciences, line of research III.23, and Academic D. I. Mendeleev Fund Program of Tomsk State University (Project No. 8.1.01.2018). The support by the Saint Petersburg State University for scientific investigations (Grant ID 40990069) and Russian Foundation for Basic Research - RFBR (Project No. 18-52-06009) are also acknowledged. T. V. Menshchikova acknowledges support by the Ministry of Education and Science of the Russian Federation within the framework of the governmental program Megagrants (State Task No. 3.8716.2017/P220 [3.8716.2017/9.10]).
[1] A. Kirilyuk, A. V. Kimel, and T. Rasing, Rev. Mod. Phys. 82, 2731 (2010).

[2] D. Pesin and A. H. MacDonald, Nat. Mater. 11, 409 (2012).

[3] J. Sanchez-Barriga, E. Golias, A. Varykhalov, J. Braun, L. V. Yashina, R. Schumann, J. Minar, H. Ebert, O. Kornilov, and O. Rader, Phys. Rev. B 93, 155426 (2016).

[4] K. C. Nowack, F. H. L. Koppens, Yu. V. Nazarov, and L. M. K. Vandersypen, Science 318, 1430 (2007).

[5] A. M. Kamerbeek, P. Högl, J. Fabian, and T. Banerjee, Phys. Rev. Lett. 115, 136601 (2015).

[6] L. P. Yang and C. P. Sun, Eur. Phys. J. B 88, 35 (2015).

[7] B. A. Bernevig, J. Orenstein, and S.-C. Zhang, Phys. Rev. Lett. 97, 236601 (2006).

[8] J. D. Koralek, C. P. Weber, J. Orenstein, B. A. Bernevig, S. C. Zhang, S. Mack, and D. D. Awschalom, Nature (London) 458, 610 (2009).
[9] M. P. Walser, C. Reichl, W. Wegscheider, and G. Salis, Nat Phys. 8, 757 (2012).

[10] M. Kohda, V. Lechner, Y. Kunihashi, T. Dollinger, P. Olbrich, C. Schönhuber, I. Caspers, V. V. Bel'kov, L. E. Golub, D. Weiss, K. Richter, J. Nitta, and S. D. Ganichev, Phys. Rev. B 86, 081306(R) (2012).

[11] J. Ishihara, Y. Ohno, and H. Ohno, Appl. Phys. Express 7, 013001 (2014).

[12] A. Sasaki, S. Nonaka, Y. Kunihashi, M. Kohda, T. Bauernfeind, T. Dollinger, K. Richter, and J. Nitta, Nat. Nanotechnol. 9, 703 (2014).

[13] C. Schönhuber, M. P. Walser, G. Salis, C. Reichl, W. Wegscheider, T. Korn, and C. Schüller, Phys. Rev. B 89, 085406 (2014).

[14] Y. S. Chen, S. Fält, W. Wegscheider, and G. Salis, Phys. Rev. B 90, 121304(R) (2014). 
[15] K. Yoshizumi, A. Sasaki, M. Kohda, and J. Nitta, Appl. Phys. Lett. 108, 132402 (2016).

[16] Moh A. U. Absor, H. Kotaka, F. Ishii, and M. Saito, Appl. Phys. Express 7, 053002 (2014).

[17] Moh A. U. Absor, H. Kotaka, F. Ishii, and M. Saito, Appl. Phys. Express 8, 073006 (2015).

[18] N. Yamaguchi and F. Ishii, Appl. Phys. Express 10, 123003 (2017).

[19] L. L. Tao and E. Y. Tsymbal, Nat. Commun. 9, 2763 (2018).

[20] H. Lee, J. Im, and H. Jin, arXiv:1712.06112 (2018).

[21] Q. N. Xu, Z. D. Song, S. M. Nie, H. M. Weng, Z. Fang, and X. Dai, Phys. Rev. B 92, 205310 (2015).

[22] D. Takane, Z. W. Wang, S. Souma, K. Nakayama, C. X. Trang, T. Sato, T. Takahashi, and Yoichi Ando, Phys. Rev. B 94, 121108(R) (2016).

[23] C. Chen, X. Xu, J. Jiang, S. C. Wu, Y. P. Qi, L. X. Yang, M. X. Wang, Y. Sun, N. B. M. Schroter, H. F. Yang, L. M. Schoop, Y. Y. Lv, J. Zhou, Y. B. Chen, S. H. Yao, M. H. Lu, Y. F. Chen, C. Felser, B. H. Yan, Z. K. Liu, and Y. L. Chen, Phys. Rev. B 95, 125126 (2017).

[24] R. Lou, J. Z. Ma, Q. N. Xu, B. B. Fu, L. Y. Kong, Y. G. Shi, P. Richard, H. M. Weng, Z. Fang, S. S. Sun, Q. Wang, H. C. Lei, T. Qian, H. Ding, and S. C. Wang, Phys. Rev. B 93, 241104(R) (2016).

[25] L. M. Schoop, M. N. Ali, C. Straer, A. Topp, A. Varykhalov, D. Marchenko, V. Duppel, S. S. P. Parkin, B. V. Lotsch, and C. R. Ast, Nat. Commun. 7, 11696 (2016).

[26] M. M. Hosen, K. Dimitri, A. Aperis, P. Maldonado, I. Belopolski, G. Dhakal, F. Kabir, C. Sims, M. Z. Hasan, D. Kaczorowski, T. Durakiewicz, P. M. Oppeneer, and M. Neupane, Phys. Rev. B 97, 121103(R) (2018).

[27] A. Topp, J. M. Lippmann, A. Varykhalov, V. Duppel, B. V Lotsch, C. R. Ast, and L. M. Schoop, New J. Phys. 18, 125014 (2016).

[28] M. M. Hosen, K. Dimitri, I. Belopolski, P. Maldonado, R. Sankar, N. Dhakal, G. Dhakal, T. Cole, P. M. Oppeneer, D. Kaczorowski, F. C. Chou, M. Z. Hasan, T. Durakiewicz, and M. Neupane, Phys. Rev. B 95, 161101(R) (2017).
[29] A. Topp, R. Queiroz, A. Grüneis, L. Müchler, A. W. Rost, A. Varykhalov, D. Marchenko, M. Krivenkov, F. Rodolakis, J. L. McChesney, B. V. Lotsch, L. M. Schoop, and C. R. Ast, Phys. Rev. X 7, 041073 (2017).

[30] Y. Kunihashi, M. Kohda, H. Sanada, H. Gotoh, T. Sogawa, and J. Nitta, Appl. Phys. Lett. 100, 113502 (2012).

[31] N. Kumar, K. Manna, Y. P. Qi, S. C. Wu, L. Wang, B. H. Yan, C. Felser, and C. Shekhar, Phys. Rev. B 95, 121109(R) (2017).

[32] H. Iwasawa, K. Shimada, E. F. Schwier, M. Zheng, Y. Kojima, H. Hayashi, J. Jiang, M. Higashiguchi, Y. Aiura, H. Namatame, and M. Taniguchi, J. Synchrotron Rad. 24, 836 (2017).

[33] H. Hayashi, K. Shimada, J. Jiang, H. Iwasawa, Y. Aiura, T. Oguchi, H. Namatame, and M. Taniguchi, Phys. Rev. B 87, 035140 (2013).

[34] T. Okuda, K. Miyamoto, A. Kimura, H. Namatamea, and M. Taniguchi, J. Electron Spectros. Relat. Phenom. 201, 23 (2015).

[35] M. Sakano, K. Okawa, M. Kanou, H. Sanjo, T. Okuda, T. Sasagawa, and K. Ishizaka, Nat. Commun. 6, 8595 (2015).

[36] B. J. Feng, Y. H Chan, Y. Feng, R. Y. Liu, M. Y. Chou, K. Kuroda, K. Yaji, A. Harasawa, P. Moras, A. Barinov, W. Malaeb, C. Bareille, T. Kondo, S. Shin, F. Komori, T. C. Chiang, Y. G. Shi, and I. Matsuda, Phys. Rev. B 94, 195134 (2016).

[37] P. E. Blöchl, Phys. Rev. B 50, 17953 (1994).

[38] G. Kresse and D. Joubert, Phys. Rev. B 59, 1758 (1999).

[39] G. Kresse and J. Furthmüller, Comput. Mater. Sci. 6, 15 (1996).

[40] J. P. Perdew, K. Burke, and M. Ernzerhof, Phys. Rev. Lett. 77, 3865 (1996).

[41] D. D. Koelling and B. N. Harmon, J. Phys. C 10, 3107 (1977).

[42] M. P. L. Sancho, J. M. L. Sancho, J. M. L. Sancho, and J. Rubio, J. Phys. F 15, 851 (1985).

[43] J. Henk and W. Schattke, Comput. Phys. Commun. 77, 69 (1993).

[44] T. Ozaki, Phys. Rev. B 67, 155108 (2003).

[45] See Supplemental Material at http://link.aps.org/supplemental/ 10.1103/PhysRevB.100.205140 for details on the calculated orbital-resolved Fermi surface map and electronic structures along the high symmetry lines.

[46] I. A. Nechaev, P. M. Echenique, and E. V. Chulkov, Phys. Rev. B 81, 195112 (2010). 